\title{
FERROMAGNETIC RESONANCE IN VERY FINE Co PARTICLES FORMING GRANULAR Co-Cu FILMS
}

\author{
J. DUBowIK \\ Institute of Molecular Physics, Polish Academy of Sciences \\ Smoluchowskiego 17, 60-179 Poznań, Poland \\ AND YU.V. KuDRYAVTSEV \\ Institute of Metal Physics, National Academy of Sciences of Ukraine \\ Vernadskogo 36, $252142 \mathrm{Kiev}$, Ukraine \\ Ferromagnetic resonance was investigated in heterogeneous $\mathrm{Co}-\mathrm{Cu}$ thin \\ films consisting of randomly oriented fine $\mathrm{Co}$ particles embedded in $\mathrm{Cu}$ ma- \\ trix. The experimental spectra are modelled using dynamic susceptibility \\ approach assuming a broad distribution in particle sizes and dipolar inter- \\ actions between particles.
}

PACS numbers: $76.50 .+\mathrm{g}, 75.50 . \mathrm{Tt}$

\section{Introduction}

Fine magnetic particles have been investigated with ferromagnetic resonance (FMR) for many years [1]. With the growing interest in giant magnetoresistance in granular materials [2], FMR has been proved useful for revealing important information on the size and shape of particles [3]. There are several aspects which are important in description of FMR response in fine particle assemblies: (i) thermally activated magnetization reversal, i.e., superparamagnetism; (ii) magnetic anisotropies; (iii) interparticle interactions and (iv) particle size distribution.

The essential factor which distinguishes FMR in fine magnetic particles from that in polycrystals is their superparamagnetic behaviour. If the sizes of particles are so small that thermal fluctuations of the magnetic moments temporarily disturb their equilibrium configuration, the "static" picture is no longer valid and a statistical approach is needed. Our experimental data will be interpreted in the framework of such an approach [4] extended to take into account dipolar interactions between particles as well as their size distribution. The description of FMR in fine particles will be limited here to the main concepts. A full discussion can be found in the original paper [4].

Let us consider a fine particle of volume $v$, magnetic moment $\mu=M v$ and the uniaxial anisotropy $K_{\mathrm{u}}$ which is subjected to a slowly varying magnetic field 
$H$ at temperature $T$. In addition, a small alternating field $h$ of frequency $\omega / 2 \pi$ is applied perpendicular to $H$. In the statistical approach, the dynamics of $\mu$ will be described by a kinetic equation of the Fokker-Planck type in which thermal fluctuations are rendered by a rotary distribution function with $\xi_{0}=\mu \omega / \gamma k T$ (a reduced Zeeman energy for $H_{0}=\omega / \gamma$ ) as a parameter. This approach enables the FMR response of a single particle to be determined by the imaginary part of complex dynamic susceptibility $\chi_{+}$

$$
\chi_{+}(H)=\frac{\mu^{2}}{k T} \frac{R}{\lambda+2 \mathrm{i} \omega \tau},
$$

where + symbol stands for a clockwise circular $h$ polarization and $\tau$ is the relaxation time related to the dimensionless damping constant $\alpha$ of the Landau-Lifshitz equation of motion. The diagonal elements $R$ and $\lambda$ of the matrix coefficients are dependent on the orientation of a particle so that in order to calculate the average dynamic susceptibility of an assembly of particles, one should know the angulair dispersion of their easy axes (i.e., their texture). We assume a random distribution of the easy axes.

Finally, a distribution of the particle sizes should also be considered. In real systems there is a size distribution so that the resultant shape of FMR absorption is given by

$$
P(H)=\int \mathrm{d} v f(v) \operatorname{Im} \bar{\chi}_{+}(v, H),
$$

where $f(v)$ is the size distribution function.

\section{Results and discussion}

$\mathrm{Co}_{0.22} \mathrm{Cu}_{0.78}$ films were obtained by a flash evaporation onto glass substrates kept at temperatures ranged from 150 to $720 \mathrm{~K}$. Since depositing films at elevated substrate temperature causes the formation of granular magnetic systems consisting of single-domain ferromagnetic particles embedded in a metallic medium [6], such a procedure enabled us to control the size of Co particles in a wide range. Films were examined with transmission electron microscopy (TEM). The magnetization and hysteresis loop were measured using a Faraday balance and vibrating sample magnetometer (VSM). FMR measurements were performed at $9.2 \mathrm{GHz}$ with the applied field both parallel and perpendicular to the plane of the samples. Our TEM studies led us to the important finding that all precipitated particles exhibit random orientation.

To evaluate the parameters characterizing Co particles, we used static magnetic measurements. Applying the standard fitting procedure to experimental magnetization curves [5] with the log-normal size distribution, we evaluated average volume $\bar{v}$ and its standard deviation $\sigma$ (see Table). However, the fitting is not very sensitive to the shape of the distribution function. Magnetic measurements revealed small coercivity $H_{\mathrm{c}}$ increasing with $\bar{v}$ (Table).

Hence, the Co-Cu films consist of fine Co particles broadly spread in sizes and randomly oriented in $\mathrm{Cu}$ matrix. Since Co particles are $f c c$ in structure, the magnetocrystalline anisotropy is rather small [7]. However, even a $3 \%$ departure from sphericity gives rise to a substantial uniaxial anisotropy $K_{\mathrm{u}}=10^{5} \mathrm{erg} / \mathrm{cm}^{3}$ and thus we assume that Co particles exhibit the uniaxial anisotropy. 
TABLE

Basic parameters characterizing $\mathrm{Co}-\mathrm{Cu}$ granular films produced at the substrate temperature $T_{\mathrm{s}}$.

\begin{tabular}{c|c|c|c|c|c|c|c|r}
\hline \hline $\begin{array}{c}\text { Sample } \\
\text { No. }\end{array}$ & $\begin{array}{c}T_{\mathrm{S}} \\
{[\mathrm{K}]}\end{array}$ & $\begin{array}{c}\bar{v} \\
\times 10^{20}\left[\mathrm{~cm}^{3}\right]\end{array}$ & $\sigma$ & $\xi_{0}$ & $\begin{array}{c}4 \pi M_{\text {eff }} \\
{[\mathrm{G}]}\end{array}$ & $\begin{array}{c}4 \pi M_{\text {eff }}^{\text {calc }} \\
{[\mathrm{G}]}\end{array}$ & $g$ & $\begin{array}{c}H_{\mathrm{c}} \\
{[\mathrm{Oe}]}\end{array}$ \\
\hline I & 150 & 0.3 & 1.1 & 0.3 & 200 & 300 & 2.26 & 0 \\
II & 400 & 1.3 & 1.7 & 1.4 & 530 & 800 & 2.34 & 50 \\
III & 520 & 4.6 & 1.9 & 5.0 & 1350 & 1500 & 2.26 & 50 \\
IV & 720 & 7.0 & 0.9 & 8.0 & 3010 & 3200 & 2.36 & 100
\end{tabular}

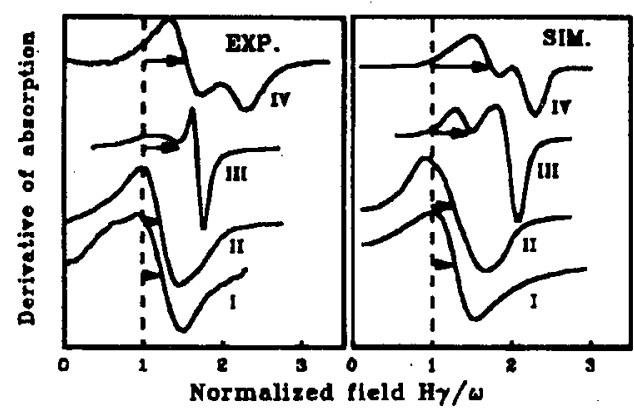

Fig. 1. X-band FMR spectra for $\mathrm{Co}-\mathrm{Cu}$ granular films taken at the perpendicular configuration (EXP.) and the results of simulation of the field derivative of FMR absorption $\mathrm{d} P / \mathrm{d} H$ (Eq. (1)) for randomly oriented Co particles with uniaxial anisotropy and a log-normal size distribution (SIM.).

The FMR spectra taken at the perpendicular configuration are shown in Fig. 1. The resonance field positions lie substantially above $x=1$ for the perpendicular configuration which is typical of planar samples. From the resonance positions at the perpendicular and in-plane configurations, an effective $\dot{g}$-factor and magnetization $4 \pi M_{\text {eff }}$ were calculated using the Kittel resonance condition for planar samples [8]. The experimental values of $4 \pi M_{\text {eff }}$ in Table are interpreted in the framework of the mean field approach for interacting fine particles which form of a thin film [9]; $4 \pi M_{\text {eff }}$ increases with increasing the mean particle volume according to

$$
4 \pi M_{\mathrm{eff}}=4 \pi p M \frac{\left[1-3 L\left(\xi_{0}\right) / \xi_{0}\right]}{L\left(\xi_{0}\right)},
$$

where $p$ is the volumetric filling factor ( $p=0.2$ in our case) of Co particles assumed to be nearly spherical and $L\left(\xi_{0}\right)$ is the Langevin function [4] which accounts for thermal fluctuations. Results of calculations according to Eq. (3) are in fairly good agreement with the experimental ones if we assume $M=1450 \mathrm{G}$ [7] and the appropriate average particle volume for each sample.

The shapes of FMR spectra are quite regular for smaller particles, becoming more and more distorted for larger particles. For the larger particles, the rise 
of the derivative of FMR absorption is gentler on the low field side, the fall on the high field side being steeper. The simulated spectra according to the field derivative of $P(H)$ (Eq. (2)) are presented in Fig. 1. The evolution of the line shapes and their positions are fairly well reproduced assuming the following parameters: $M=1450 \mathrm{G}, K_{\mathrm{u}}=10^{5} \mathrm{erg} / \mathrm{cm}^{3}, T=300 \mathrm{~K}, \alpha=0.05$, and $\bar{v}$ taken from Table. It was found that the mean size mainly affects the line position described by Eq. (3) while the shapes are primary influenced by the shape of size distribution function. Though we did not attempt to achieve the best fits, it appears that the main features of the spectra in the Co-Cu granular films can be reproduced assuming slightly modified log-normal size distribution with the $\sigma$ values taken from Table. Therefore, peculiarities of the size distribution function seem to play the decisive role in building up the shape of FMR spectra of fine particle systems.

In conclusion, FMR spectra of fine $\mathrm{Co}$ particles embedded in $\mathrm{Cu}$ thin film matrix have been satisfactorily interpreted in the framework of theory of FMR extended by a reasonable assumption of distribution of the particle sizes and dipolar interaction between them. It has been shown that the line position is mainly influenced by the interactions between particles while the line shape results mainly from the shape of size distribution function $f(v)$.

\section{References}

[1] D.M.S. Bagguley, Proc. Phys. Soc. A 66, 765 (1953).

[2] S.S.P. Parkin, in: Ultrathin Magnetic Structures II, Eds. B. Heinrich, J.A.C. Bland, Springer, Berlin 1994, p. 181.

[3] M. Rubinstein, B.N. Das, N.C. Koon, D.B. Chrisey, J. Horwitz, Phys. Rev. B 50, 184 (1994).

[4] Yu.L. Raikher, V.I. Stepanov, Phys. Rev. B 50, 6250 (1994).

[5] J.T. Richardson, J. Appl. Phys. 49, 1781 (1978).

[6] J.Q. Xiao, J.S. Jiang, L. Chien, Phys. Rev. Lett. 68, 3749 (1992).

[7] G. Abersfelder, K. Noack, K. Stierstadt, J. Schelten, W. Schmatz, Philos. Mag. B 41, 519 (1980).

[8] C. Kittel, Phys. Rev. 73, 155 (1948).

[9] J. Dubowik, Phys. Rev. B 54, 1088 (1996). 